\title{
Role of multi-parametric MRI in the differentiation between mucinous breast carcinoma and fibroadenoma
}

\author{
Dalia Bayoumi ${ }^{1}$, Amal Sakrana ${ }^{1}$ Ebrahim Abdelhalim ${ }^{2}$ and Eman Alnaghy ${ }^{1^{*}}$ (D)
}

\begin{abstract}
Background: The purpose of this study was to evaluate diagnostic performance of dynamic contrast-enhanced magnetic resonance imaging (DCE-MRI) and functional MRI (diffusion and MR spectroscopy) using the latest MRI breast imaging reporting and data system (MRI-BIRADS) descriptors and non-BIRADS items for differentiation of mucinous breast carcinomas from fibroadenomas.

Methods: We included 19 cases of mucinous breast carcinoma and 37 cases of fibroadenoma. DCE-MRI, Diffusionweighted MR imaging (DWI) and multi-voxel MR spectroscopy (MRS) were done, MRI-BIRADS (5th edition) analysis of the lesions was performed, and histopathological examination of all cases was done.

Results: According to univariate analysis, mucinous carcinoma was frequently detected in older age-group $(\mathrm{COR}=1.4,95 \% \mathrm{Cl}=1.1-1.7$ and $p$ value $<0.001)$ and had more frequent irregular borders and non-circumscribed margins than fibroadenoma ( $C O R=11.6,95 \% \mathrm{Cl}=2-66.4$ and $p$ value $=0.002)$. All mucinous carcinoma had high T2 signal. Fibroadenomas had more frequent homogenous enhancement than mucinous carcinoma; none of the fibroadenomas had rim enhancement nor enhancing internal septations; mucinous carcinoma had more frequent rim enhancement and $(n=6, p$ value $<0.001)$ and enhancing internal septations $(n=7, p$ value $<0.001)$. Fibroadenoma had frequent dark non-enhancing internal septations than mucinous carcinoma ( $p$ value $<0.001$ ). On multivariate analysis, mucinous carcinoma had significant combination of being common in older age, larger in size, with irregular shape, and non-circumscribed margins. On ADC map and MRS, mucinous carcinoma had higher ADC values and higher CHO.SNR than fibroadenoma. The mean ADC value of mucinous carcinoma was $1.3 \pm 0.1 \times 10^{-3} \mathrm{~mm}^{2} / \mathrm{s}$, which was significantly higher than that of fibroadenoma $\left(1.1 \pm 0.1 \times 10^{-3} \mathrm{~mm}^{2} / \mathrm{s}\right)$, with $p=0.002$. Also, the mean CHO.SNR was significantly higher in mucinous carcinoma (3.1 \pm 0.8$)$ than fibroadenoma $(0.8 \pm 0.5)$ with $p$ value $<0.001$. According to our results, the presence of type 3 (washout curve), dark internal septations, non-circumscribed margins and irregular shape of the lesion showed the highest sensitivity and accuracy for differentiation of mucinous carcinomas and fibroadenomas $(100,78.6 \%),(89.4,82 \%),(86.5,80.3 \%)$ and $(81,71.4 \%)$, respectively.
\end{abstract}

Conclusion: The combined use of DCE-MRI, DW-MRI and MRS with breast MRI-BIRADS descriptors and non-BIRADS items increases the diagnostic accuracy for differentiation of mucinous carcinomas from fibroadenomas.

Keywords: Breast MRI, Fibroadenoma, Mucinous carcinoma, Diffusion, MR spectroscopy

\footnotetext{
*Correspondence: emnaghi@hotmail.com

1 Department of Diagnostic Radiology, Mansoura Faculty of Medicine,

Mansoura University, Mansoura, Egypt

Full list of author information is available at the end of the article
}

\begin{abstract}
Background
Mucinous (colloid) carcinoma of the breast is uncommon type of infiltrating breast carcinomas representing $1-4 \%$ of all breast cancers. It has a slow rate of growth with good prognosis. Radiologically mucinous breast
\end{abstract}


carcinoma often mimics fibroadenomas, so it may be associated with delayed diagnosis. Mucinous carcinomas are classified into pure and mixed subtypes; pure mucinous carcinomas have favorable prognosis, while mixed carcinomas generally have poorer prognosis similar to invasive ductal carcinomas [1].

According to the World Health Organization (WHO), the pure type consists exclusively of tumor tissue with extracellular mucin production, while mixed type is defined as tumors containing $50-90 \%$ mucinous component mixed with infiltrating ductal epithelial components. Pure mucinous carcinomas show less aggressive behavior with less axillary lymph nodes metastasis and can be managed with less invasive surgery. Positive nodal status is the most significant predictor of worse prognosis. Mixed mucinous carcinomas with prominent invasive components are different from fibroadenomas and have clear radiological diagnostic features of malignancy, while the pure type shows benign findings similar to fibroadenoma on both mammography (MMG) and ultrasonography (US) [2].

Fibroadenomas are fibroepithelial breast lesions mainly occurring in young females in reproductive years and result from abnormalities of normal development and involution of breast tissue. Typical lesions are oval, rounded or lobular with internal septations. Multiple, giant and juvenile lesions may also be detected. Differentiation of fibroadenoma from other similarly appearing lesion is mandatory, and biopsy may be required in such cases when there is poor patient compliance with follow-up visits [3].

Recently, DCE-MRI has been widely used for detection and characterization of breast cancer; lesions may be assessed by using either their morphologic features, their kinetics or time-intensity curve characteristics; however, the best results can be achieved by the combination of both methods [4].

Noninvasive functional MRI assessment of breast lesions is achieved by using DWI and MRS. DWI can assess tissue properties depending on difference in their water molecular motion along multiple spatial directions, which is quantified by measurement of apparent diffusion coefficient (ADC). Malignant lesions show restricted diffusion with decreased ADC values compared with benign lesions. Proton MR spectroscopy can detect biochemical tissue properties. Total choline (t Cho) resonance at 3.14-3.34 ppm has been associated with oncogenesis and tumor progression and detected in malignant breast lesions as a result of complex metabolism $[5,6]$.

The aim of this study was to evaluate diagnostic performance of DCE-MRI, DW-MRI and MRS in addition to using the latest breast MRI-BIRADS descriptors and
non-BIRADS items for differentiation of mucinous breast carcinomas from fibroadenomas.

\section{Methods \\ Patients}

In this study, 56 consecutive patients were included: 19 patients with mucinous breast carcinoma and 37 patients with breast fibroadenoma in the period from March 2017 to September 2020, at our Radiology Department. Retrospective analysis was performed for all patients who underwent pre-contrast MRI, DCE-MRI, DWI and MRS of the breast. Inclusion criteria included untreated pathologically diagnosed patients with mucinous carcinoma and fibroadenoma who were referred to MR imaging for lesion characterization or as a part of clinical routine work. Patients were excluded if the lesion was not assessed by histopathology (we only included pathologically proved lesions), and patients with any contraindications to MRI were also excluded. We obtained institutional review board approval, and informed consents from the patients were waived because this is a retrospective study.

\section{MRI protocol}

All MRI studies were performed with 1.5-T MR imaging unit (Philips, Ingenia). MR imaging pre-contrast sequences included axial T1- and T2-weighted images, and STIR images of $3 \mathrm{~mm}$ slice thickness, inter-slice gap $1 \mathrm{~mm}$, a field of view (FOV) $20-30 \mathrm{~cm}$ and acquisition matrix of $256 \times 224$ were obtained. Gadolinium chelates were injected at a dose of $0.2 \mathrm{mmol} / \mathrm{kg}$ using automated injector, and images (in three phases) with subtraction in the axial plane T1-weighted MR images $(\mathrm{TR} / \mathrm{TE}=400$ 575/13-15 ms) with fat suppression were obtained. Fat suppression was accomplished with a frequency-selective pre-saturation pulse. Diffusion-weighted images were obtained using a multi-slice, spin echo, echo planar image sequence. A set of multiple axial scans were obtained. The imaging parameters were as follows: $\mathrm{TR}=10.000 \mathrm{~ms}$, $\mathrm{TE}=108 \mathrm{~ms}, \quad \mathrm{NEX}=12, \quad$ bandwidth $=300 \mathrm{kHz}$, matrix $=256 \times 128, \quad F O V=20-30 \mathrm{~cm}$, section thickness $=5 \mathrm{~mm}$ and inter-slice gap $=1 \mathrm{~mm}$. The diffusion gradients were applied in three orthogonal directions $(x$, $y$ and $z$ ). Diffusion images were acquired with a diffuse b factor of 0,600 and $1000 \mathrm{~mm}^{2} / \mathrm{s}$, and ADC maps were reconstructed. Multi-voxel MR spectroscopy was done to all patients using point-resolved spectroscopy sequence (PRESS), single 10-mm-thick sagittal section, followed by automatic and manual shimming of water signal; simultaneous water and lipid suppression was done using MEGA pulses. The following parameters were used: TR $2000 \mathrm{~ms}$ and TE $272 \mathrm{~ms}$, voxel size $5 \times 5 \times 10 \mathrm{~mm}$, FOV $80 \times 80 \mathrm{~mm}$, and scan resolution $16 \times 16 \mathrm{~mm}$. To improve 
the SNR, four acquisitions were done with 12-min total data acquisition time. The CSI grid was carefully placed to allow maximum lesion coverage.

\section{Image analysis}

Using secondary workstation (Philips Advantage Windows Workstation with functional tool software), image analysis was performed in a consensus by two breast imaging expert radiologists with 10 and 8 years of experience (B.D and A.E), respectively, who were blinded to clinical and histopathological data. Subtraction images were obtained, and time-to-signal intensity curves were created by placing the ROI at the most enhancing part of the lesions. Curves were classified into type 1 (persistent), type 2 (plateau) and type 3 (washout).

- Type 1 curve:

- Progressive or persistent enhancement, increased signal intensity with time.

- Mainly detected in benign lesions.

- Type 2 curve:

- Initial uptake followed by plateau.

- Intermediate suspicion for malignancy.

- Type 3 curve:

- Rapid uptake followed by washout.

- Intermediate suspicion for malignancy.

The internal enhancement criteria were evaluated in the delayed phase of DCE-MRI which is more appropriate than the early phase because mucinous carcinomas and fibroadenomas tend to have persistent enhancement. ADC values were automatically calculated at ADC maps by manually tracing a ROI within the boundaries of the lesion using electronic cursor at all sections of the tumor, and mean ADC value of the tumor was calculated. The two radiologists retrospectively re-analyzed the lesions according to imaging criteria of MRI-BIRADS v 2013 [7]. Lesion analysis was performed using MRI-BIRADS (5th edition) descriptors and non-BIRADS items. MRI-BIRADS descriptors included tumor shape (oval, rounded and irregular), margins (circumscribed and noncircumscribed), enhancement pattern (homogenous, heterogeneous, rim enhancement and enhancing internal septations), dark internal septations (present or absent) and dynamic curve (type 1, 2 and 3). Non-BIRADS items included age, tumor size, T2 signal (high and not high) and ADC value and total choline signal. Lesion analysis was correlated with histopathological results. Final histopathological diagnosis of the lesions was made by using 14 Gauge core needle biopsy or after surgical excision. (All histopathologically proven mucinous carcinoma cases underwent excision.) The pathological type of mucinous carcinoma was classified according to WHO classification into pure and mixed types.

\section{Statistical analysis and data interpretation}

Data were analyzed using IBM SPSS Corp. Released 2013 IBM SPSS Statistics for Windows, Version 22.0. Qualitative data were described using number and percent. Quantitative data were described using mean and standard deviation for parametric data after testing normality using Kolmogorov-Smirnov test. Significance of the obtained results was judged at the 0.05 level. Qualitative data analysis was performed using chi-square test for comparison of two or more groups and Fisher's exact test. Quantitative data analysis between groups was performed using Student's t test to compare two independent groups. Diagnostic accuracy was detected using ROC curve analysis; sensitivity and specificity were detected from the curve; and PPV, NPV and accuracy were also calculated through cross-tabulation. Binary stepwise logistic regression analysis was performed for prediction of independent variables of binary outcome. Significant predictors in the univariate analysis were entered into regression model using forward Wald method. Adjusted odds ratios and their 95\% confidence interval were calculated.

\section{Results}

This study included 56 consecutive patients: 19 patients with mucinous breast carcinoma and 37 patients with breast fibroadenoma; all were females with mean age 45 $y$ for mucinous carcinoma and 32 y for fibroadenoma. Patients were presented with palpable mass $(n=27)$, mastalgia $(n=25)$ and incidentally discovered on screening $(n=13)$. The pathological type of mucinous carcinoma was classified according to WHO classification into pure type $(n=11)$ and mixed type $(n=8)$. Univariate analysis revealed significant difference in the mean age between mucinous carcinoma $(45.8 \pm 6.1)$ and fibroadenoma $(31.9 \pm 6.7)(\mathrm{COR}=1.4,95 \% \mathrm{CI}=1.1-1.7$ and $p$ value $<0.001)$. The shape of mucinous carcinomas and fibroadenomas was oval $(n=9$ and 30$)$, rounded $(n=3$ and 5) and irregular ( $n=7$ and 2), respectively; mucinous carcinomas had more frequent irregular shape than fibroadenomas $(\mathrm{COR}=11.6,95 \% \mathrm{CI}=2-66.4$ and $p$ value $=0.002$ ).

Circumscribed margin was detected in 6 mucinous carcinoma cases and 32 fibroadenoma cases, while non-circumscribed margin was detected in 13 cases and 5 cases of mucinous carcinoma and fibroadenoma, respectively 
$(\mathrm{COR}=13.8,95 \% \mathrm{CI}=3.5-53.5$ and $p$ value $=0.003)$. The mean major (long) diameter of mucinous carcinomas was $(32.1 \pm 5.6 \mathrm{~mm})$ and for fibroadenoma $(22.2 \pm 7.3 \mathrm{~mm})(\mathrm{COR}=1.2,95 \% \mathrm{CI}=1.1-1.4$ and $p$ value less than 0.001), and the mean short diameter of mucinous carcinoma was $(21.9 \pm 6.02 \mathrm{~mm})$ and for fibroadenoma was $(13.5 \pm 5.2 \mathrm{~mm})(\mathrm{COR}=1.37,95 \% \mathrm{CI}=1.1-1.6$ and $p$ value less than 0.001 ). All mucinous carcinomas had high signal on T2-WI, while fibroadenomas had high T2 signal $(n=27)$ and not high signal $(n=10)$. Enhancement of mucinous carcinoma and fibroadenoma was homogenous $(n=2$ and $34, p$ value $=0.003)$, heterogeneous $(n=4$ and 3), rim enhancement $(n=6$ and $0, p$ value $<0.001)$ and enhancing internal septations $(n=7$ and $0, p$ value $<0.001$ ), respectively. Dark non-enhanced internal septations were present in 29 (78.4\%) fibroadenoma cases, while it was seen in only two $(10.5 \%)$ mucinous carcinoma cases, $p$ value $<0.001$. The mean ADC value of mucinous carcinoma was $1.3 \pm 0.18 \times 10^{-3} \mathrm{~mm}^{2} / \mathrm{s}$ which was significantly higher than that of fibroadenoma $\left(1.19 \pm 0.1 \times 10^{-3} \mathrm{~mm}^{2} / \mathrm{s}\right)$ with $p=0.002$. Also, the mean CHO.SNR was significantly higher in mucinous carcinoma $(3.1 \pm 0.8)$ than fibroadenoma $(0.8 \pm 0.52)$ with $p$ value $<0.001$.

On multivariate analysis age on presentation had statistically significant difference between mucinous carcinomas and fibroadenomas $(\mathrm{AOR}=1.4,95 \% \mathrm{CI}=1.1-1.7$ and $p$ value $=0.003$ ). Neither the long lesion diameter $(\mathrm{AOR}=1,95 \% \mathrm{CI}=0.8-1.2$ and $p$ value $=0.6)$ nor the short diameter $(\mathrm{AOR}=1.3,95 \% \mathrm{CI}=0.9-1.9$ and $p$ value $=0.07$ ) had significant statistical results in multivariate analysis. As regard the shape, neither the oval nor the round shape $(\mathrm{AOR}=2.1,95 \% \mathrm{CI}=0.4-9.6$ and $p=0.3$ ) had significant statistical results; the irregular shape had significant statistical results $(A O R=13.6,95 \%$ $\mathrm{CI}=2.5-72.2$ and $p$ value $=0.004)$. Non-circumscribed margins had significant statistical results $(\mathrm{AOR}=12.6$, $95 \% \mathrm{CI}=1.1-140.5$ and $p$ value $=0.004)$. Overall percent predicted was $91.9 \%$ (Table 1).

Sensitivity, specificity, PPV, NPV and accuracy of the most significant diagnostic MR features for differentiation of mucinous carcinomas and fibroadenomas are listed in Table 2.

ROC curve analysis was performed to detect the validity of ADC and CHO.SNR in differentiating fibroadenoma from mucinous tumors (Fig. 1). As regard the ADC value, AUC was 0.78 and the ADC cutoff value for differentiating mucinous carcinoma from fibroadenoma was 1.3 below which was considered as fibroadenoma and values at or above were considered mucinous carcinoma. The sensitivity was $70.3 \%$, specificity $=64 \%, P P V=74.3 \%$, $\mathrm{NPV}=59.3 \%$ and accuracy $=67.7 \%$. As regard the CHO.SNR, AUC was 1 and CHO.SNR cutoff value for differentiating mucinous carcinoma from fibroadenoma was 1.97 values, below which were considered as fibroadenoma and values at or above were considered mucinous carcinoma. The sensitivity was $100 \%$, specificity $=96 \%$, $\mathrm{PPV}=97.4 \%, \mathrm{NPV}=100 \%$ and accuracy $=98.4 \%$. In combined use of CHO.SNR and ADC value, AUC was 1 , and the sensitivity, specificity, PPV, NPV and accuracy reached $100 \%$ (Table 3 ).

\section{Discussion}

According to univariate analysis, mucinous carcinoma was frequently detected in older age-group, was larger than fibroadenoma on presentation and had more frequent irregular borders and non-circumscribed margins, and all mucinous carcinoma had high T2 signal. Fibroadenomas had more frequent homogenous enhancement than mucinous carcinoma, none of the fibroadenomas had rim enhancement or enhancing internal septations, while mucinous carcinoma had frequent rim enhancement and enhancing internal septations. Fibroadenoma had frequent dark non-enhancing internal septations than mucinous carcinoma. On ADC map and MRS, mucinous carcinoma had higher ADC values and higher CHO.SNR than fibroadenoma. On multivariate analysis, mucinous carcinoma had a significant combination of being common in older age, larger in size, with irregular shape, and non-circumscribed margins.

According to our results, the presence of type 3 (washout curve), dark internal septations, non-circumscribed margins and irregular shape of the lesion showed the highest sensitivity and accuracy for differentiation of mucinous carcinomas and fibroadenomas (100, 78.6\%), (89.4, 82\%), (86.5, 80.3\%) and (81, 71.4\%), respectively.

The previous study performed by Igarashi et al. [2] showed that combination of irregular margin and delayed heterogeneous enhancement showed the highest sensitivity and accuracy $(96.3 \%, 87.8 \%)$ for differentiation of mucinous carcinomas and fibroadenomas.

Regarding lesion shape and margins: Mucinous carcinomas had more frequent irregular shape and non-circumscribed margins $(13 / 19,68.4 \%)$ than fibroadenomas). In agreement with our results, Igarashi et al. [2] reported that some mucinous carcinomas showed non-circumscribed margins in the univariate analysis $(11 / 27,41 \%)$. Margin irregularity is more characteristic of mixed mucinous carcinomas and is attributed to fibrotic and infiltrative nature of their non-mucinous component.

Regarding lesion enhancement: In our study, 34 fibroadenoma cases (91.9\%) had homogenous enhancement and only three cases $(8.1 \%)$ showed heterogeneous enhancement which may be attributed to cystic changes. Patterns of mucinous tumor enhancement in dynamic study depend on the amount of mucin versus the solid 
Table 1 Association between risk factors and pathology among studied cases

\begin{tabular}{|c|c|c|c|c|c|c|}
\hline & \multicolumn{2}{|l|}{ Pathology } & \multicolumn{2}{|l|}{ Univariate analysis } & \multicolumn{2}{|c|}{ Multivariate analysis } \\
\hline & $\begin{array}{l}\text { Mucinous } \\
N=19\end{array}$ & $\begin{array}{l}\text { Fibroadenoma } \\
N=37\end{array}$ & Test of significance & $\begin{array}{l}\text { COR } \\
(95 \% \mathrm{Cl})\end{array}$ & $\mathrm{P}$ & $\begin{array}{l}\text { AOR } \\
(95 \% \mathrm{Cl})\end{array}$ \\
\hline $\begin{array}{l}\text { Age/years } \\
\text { Mean } \pm S D\end{array}$ & $45.8 \pm 6.1$ & $31.9 \pm 6.7$ & $\begin{array}{l}t=7.5 \\
p<0.001^{*}\end{array}$ & $1.4(1.1-1.7)$ & $0.003^{*}$ & $1.4(1.1-1.7)$ \\
\hline $\begin{array}{l}\text { Long diameter } \\
\text { Mean } \pm S \mathrm{SD}\end{array}$ & $32.1 \pm 5.5$ & $22.1 \pm 7.3$ & $\begin{array}{l}t=5.7 \\
p<0.001^{*}\end{array}$ & $1.2(1.13-1.46)$ & 0.6 & $1.06(0.8-1.2)$ \\
\hline $\begin{array}{l}\text { Short diameter } \\
\text { Mean } \pm S D\end{array}$ & $21.9 \pm 6$ & $13.5 \pm 5.2$ & $\begin{array}{l}t=5.7 \\
p<0.001^{*}\end{array}$ & $1.3(1.1-1.6)$ & 0.07 & $1.3(0.9-1.9)$ \\
\hline $\begin{array}{l}\mathrm{ADC}^{*} 10-3 / \mathrm{mm} \\
\text { Mean } \pm \mathrm{SD}\end{array}$ & $1.3 \pm 0.1$ & $1.1 \pm 0.1$ & $\begin{array}{l}t=3.9 \\
p=0.002^{*}\end{array}$ & 508 (undefined) & 0.9 & \\
\hline CHOSNR & $3.1 \pm 0.8$ & $0.8 \pm 0.5$ & $\begin{array}{l}t=13.5 \\
p<0.001^{*}\end{array}$ & (Undefined) & & \\
\hline \multicolumn{7}{|l|}{ mean $\pm S D$} \\
\hline \multicolumn{7}{|l|}{ Shap n (\%) } \\
\hline Oval (r) & $9(47.3)$ & $30(81.1)$ & 1 & 1 & & 1 \\
\hline Round & $3(15.7)$ & $5(13.5)$ & $x^{2}=0.7, p=0.39$ & $2(0.39-10)$ & 0.3 & $2.1(0.4-9.6)$ \\
\hline Irregular & $7(36.8)$ & $2(5.4)$ & $x^{2}=9.8, p=0.002^{*}$ & $11.6(2-66.4)$ & $0.004^{*}$ & $13.6(2.5-72.2)$ \\
\hline \multicolumn{7}{|l|}{ Margin $n(\%)$} \\
\hline Circumscribed $(r)$ & $6(31.5)$ & $32(86.5)$ & $x^{2}=17.4, p=0.003^{*}$ & 1 & $0.004^{*}$ & 1 \\
\hline Non-circumscribed & $13(68.4)$ & $5(13.5)$ & & $13.8(3.5-53.5)$ & & $12.6(1.1140 .5)$ \\
\hline \multicolumn{7}{|l|}{ T2 Signal intensity n (\%) } \\
\hline High & $19(100)$ & $27(73)$ & $x^{2}=6.2, p=0.012^{*}$ & 1 & 0.99 & \\
\hline Not high & $0(0)$ & $10(18.9)$ & & Undefined & & \\
\hline \multicolumn{7}{|l|}{ Enhancement $n(\%)$} \\
\hline Homogeneous (r) & $2(10.5)$ & $34(91.9)$ & FET, $p=0.003^{*}$ & $22.67(2.8-179.1) 1$ & 0.9 & \\
\hline Heterogeneous & $4(21)$ & $3(8.1)$ & & Undefined & & \\
\hline Rim enhancement & $6(31.5)$ & $0(0)$ & FET, $p=0.001^{*}$ & Undefined & 0.9 & \\
\hline Enhancing internal septations & $7(36.8)$ & $0(0)$ & FET, $p<0.001^{*}$ & & 0.9 & \\
\hline \multicolumn{7}{|l|}{ Dynamic curve n (\%) } \\
\hline Type 1 & $1(5.2)$ & $29(78.3)$ & $\mathrm{FET}, p=0.2$ & Undefined & & \\
\hline Type 2 & $11(57.8)$ & $8(21.6)$ & $x^{2}=2, p=0.1$ & $2.3(0.72-0.2)$ & & \\
\hline Type $3(r)$ & $7(36.8)$ & $0(0)$ & & 1 & & \\
\hline \multicolumn{7}{|l|}{ Dark internal septations n (\%) } \\
\hline Absent & $17(89.4)$ & $8(21.6)$ & $\begin{array}{l}x^{2}=23.3 \\
p<0.001^{*}\end{array}$ & $30.8(5.8-162)$ & 0.8 & $33.4(0.8-60.8)$ \\
\hline Present (r) & $2(10.5)$ & $29(78.4)$ & & 1 & & \\
\hline
\end{tabular}

Overall $\%$ predicted $=91.9 \%$

tumor tissue. Mucinous carcinoma showed homogenous enhancement in only two cases (10.5\%): predominant solid part, heterogeneous enhancement in four cases (21\%) corresponding to islands of neoplastic epithelial cells floating in mucin, and rim enhancement (Fig. 2) in six cases $(31.5 \%)$ corresponding to a peripheral distribution of tumor cells and central location of mucin, that was in consistent with a previous study [8] which reported that hypocellular pure mucinous tumors have typical pattern of gradual enhancement and hypercellular pure tumors have strong early enhancement. Gradual enhancement pattern in mucinous carcinomas is due to large amount of mucin, and diffusion of contrast throughout the stroma and around the epithelial component takes time [9].

Internal septations: Internal septations within the lesion may be either dark non-enhanced or enhanced septa. Dark non-enhanced internal septations were detected in 29 fibroadenoma cases (78.4\%) in our study; lack of enhancement in these septa is due to the presence of hypovascular collagenous bands within the lesion. While enhanced internal septations were detected in seven mucinous carcinoma cases $(36.8 \%)$ in our study with $100 \%$ specificity and $77.4 \%$ accuracy, which is 
Table 2 The most significant diagnostic MR features for differentiation of mucinous carcinomas and fibroadenomas

\begin{tabular}{llllll}
\hline & Sensitivity (\%) & Specificity (\%) & PPV (\%) & NPV (\%) & Accuracy (\%) \\
\hline Irregular shape & 81.1 & 52.6 & 76.9 & 58.8 & 71.4 \\
Non-circumscribed margin & 86.5 & 68.4 & 84.2 & 72.2 & 80.3 \\
T2 Signal intensity & 100 & 27.03 & 41.3 & 100 & 51.8 \\
Homogenous enhancement & 91.9 & 89.5 & 94.4 & 85 & 91.1 \\
Heterogeneous enhancement & 91.9 & 21.1 & 69.4 & 57.1 & 66.1 \\
Rim enhancement & 31.6 & 100 & 100 & 72.3 & 75.5 \\
Enhancing internal septations & 36.8 & 100 & 100 & 73.9 & 77.4 \\
Dynamic curve & 94.7 & 78.3 & 96.6 & 69.2 & 83.9 \\
Type 1 & 78.3 & 94.74 & 96.6 & 69.2 & 83.9 \\
Type 2 & 78.4 & 57.2 & 78.4 & 57.2 & 71.4 \\
Type 3 & 100 & 36.8 & 75.5 & 100 & 78.6 \\
Dark internal septations & 89.4 & 78.3 & 68 & 93.5 & 82.1 \\
\hline
\end{tabular}

attributed to the vascular nature of the internal fibrous bands in contrast to the non-vascular bands in fibroadenomas, this have been suggested to differentiate mucinous carcinoma from fibroadenoma in consistent with previous studies [2] which reported that enhancing septations on dynamic CE-MRI was a characteristic finding of mucinous carcinomas (Fig. 3).

\section{Dynamic curve}

In our study, type 1 and type 2 curves were detected in both fibroadenoma and mucinous carcinoma; type 1 curve was of significance only for fibroadenoma cases; it was detected in 29 (78.3\%) fibroadenoma cases with high specificity and accuracy $(96.6,83.9 \%)$. Type 3 curve was detected only in mucinous carcinoma (7 cases, $36.8 \%$ ) with high specificity and accuracy (100\% and 78.6\%). This was not in agreement with Igarashi et al. [2] who reported that there were no significant differences in the kinetic patterns between mucinous carcinoma and fibroadenoma.

In our study, morphological features assessment was predictive for differentiation between mucinous carcinoma and fibroadenoma after exclusion of type 3 curves in dynamic study as none of fibroadenomas showed type 3 curve in our results. Previous studies were in agreement; they reported that assessment of the morphological features was more predictive in differentiation between mucinous carcinoma and fibroadenoma and in diagnosis of breast cancer than was characterization of the kinetic curves $[10,11]$.

T2 signal: In our study, the lesion signal was not specific for any point of differentiation as all cases of mucinous carcinomas and 27 fibroadenoma cases had high signal on T2 WI, while for fibrous or calcified fibroadenomas (10 cases) their T2 signal was not high.

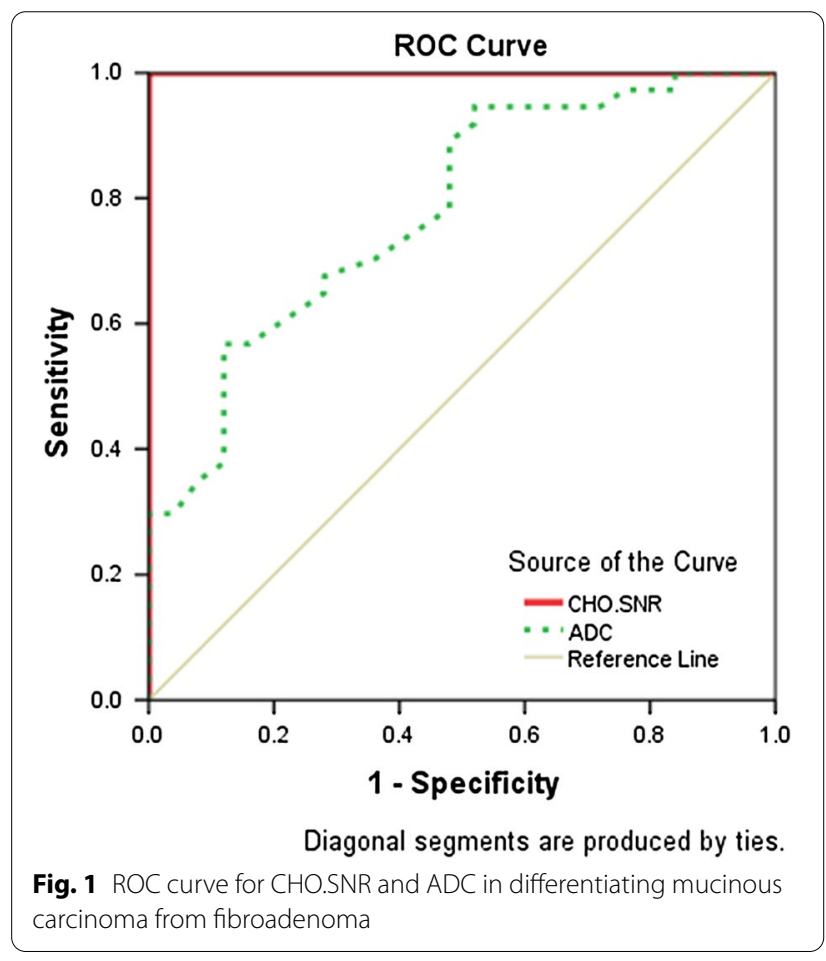

Functional assessment (Fig. 4, and Fig. 5)

Our study showed a significant difference in the mean $\mathrm{ADC}$ value between fibroadenoma and mucinous carcinoma; fibroadenomas mean ADC value was 1.19 which was significantly lower than mucinous carcinoma (1.39). This was in agreement with other previous studies [12, 13], reporting that among malignant breast tumors, mucinous carcinoma displayed the highest $\mathrm{ADC}$ values; they had a mean ADC of $1.8 \times 10^{-3} \mathrm{~mm}^{2} / \mathrm{s}$, statistically significantly greater than that of benign 
Table 3 Validity of CHO.SNR and ADC in differentiating fibroadenoma from mucinous tumors

\begin{tabular}{llllllll}
\hline & $\begin{array}{l}\text { AUC } \\
(\mathbf{9 5 \% C l )}\end{array}$ & Cutoff & Sensitivity (\%) & Specificity (\%) & PPV (\%) & NPV (\%) & Accuracy (\%) \\
\hline CHO.SNR & 1 & 1.97 & 100 & 96 & 97.4 & 100 & 98.4 \\
& $(1-1)$ & 1.32 & 70.3 & 64 & 74.3 & 59.3 & 67.7 \\
ADC $\times 10^{-3} / \mathrm{mm}$ & $\begin{array}{l}0.78 \\
(0.6-0.8)\end{array}$ & undefined & 100 & 100 & 100 & 100 & 100 \\
$\begin{array}{l}\text { Combined ADC and } \\
\text { CHO.SNR }\end{array}$ & $\begin{array}{l}1 \\
(1-1)\end{array}$ & & & & & & \\
\hline
\end{tabular}

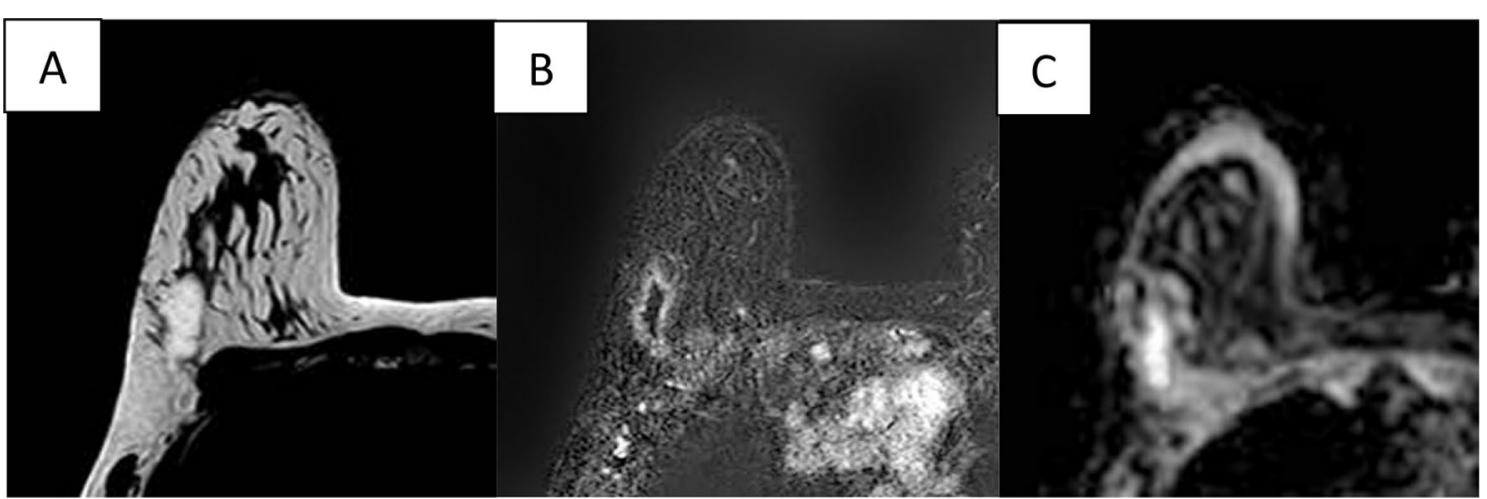

Fig. 2 A 47-year-old female with a right breast mucinous carcinoma. A Axial T2-Wl image showing a non-circumscribed lesion of high signal. B Axial postcontrast T1-WI with subtraction showing irregular marginal contrast enhancement. C Axial ADC map, the ADC value of the lesion was $1.43 \times 10^{-3} \mathrm{~mm}^{2} / \mathrm{s}$

lesions $\left(1.3 \times 10^{-3} \mathrm{~mm}^{2} / \mathrm{s}\right)$ and other malignant tumors $\left(0.9 \times 10^{-3} \mathrm{~mm}^{2} / \mathrm{s}\right)$, due to the large amount of the extracellular protein.

Others reported that mean ADC values of mucinous tumors in areas rich in tumor cells were lower than areas containing large amounts of mucin and small amounts of tumor cells and were markedly higher than solid component in conventional invasive duct carcinoma (IDC) [9].

This was not in agreement with Igarashi et al. [2] as they reported no significant difference in ADC values between fibroadenoma and mucinous carcinoma attributing this finding to T2 shine-through effect.

In our study, MRS showed significant CHO.SNR in mucinous carcinomas which was 3.1 , while in fibroadenomas it was 0.8 (Fig. 6). This was in agreement with previous studies $[14,15]$ which assessed diagnostic accuracy of MRS in differentiating benign and malignant breast lesions considering the lesion of malignant nature if the signal-to-noise ratio of choline resonance peak was equal to or greater than 2 .

\section{Limitations}

There are few limitations to this study. First was the small sample size as mucinous breast carcinoma being not a common pathological diagnosis, also multiple analysis items were included. Second, cases with typical fibroadenomas were diagnosed by US; MRI was not mandatory for diagnosis nor histopathological sampling, so a larger number of fibroadenomas are necessary to evaluate both morphologic and kinetic features for confident diagnosis. Third, there may be bias from the manual selection of region of interest. Application of automated approach would be preferred. Fourth, our study was retrospective, and some selection bias may have been present which may have limited the accuracy of results.

\section{Conclusions}

We concluded that the combined use of DCE-MRI, DWMRI and MRS in addition to using breast MRI-BIRADS descriptors and non-BIRADS items increases the diagnostic accuracy for differentiation of mucinous carcinomas from fibroadenomas. 


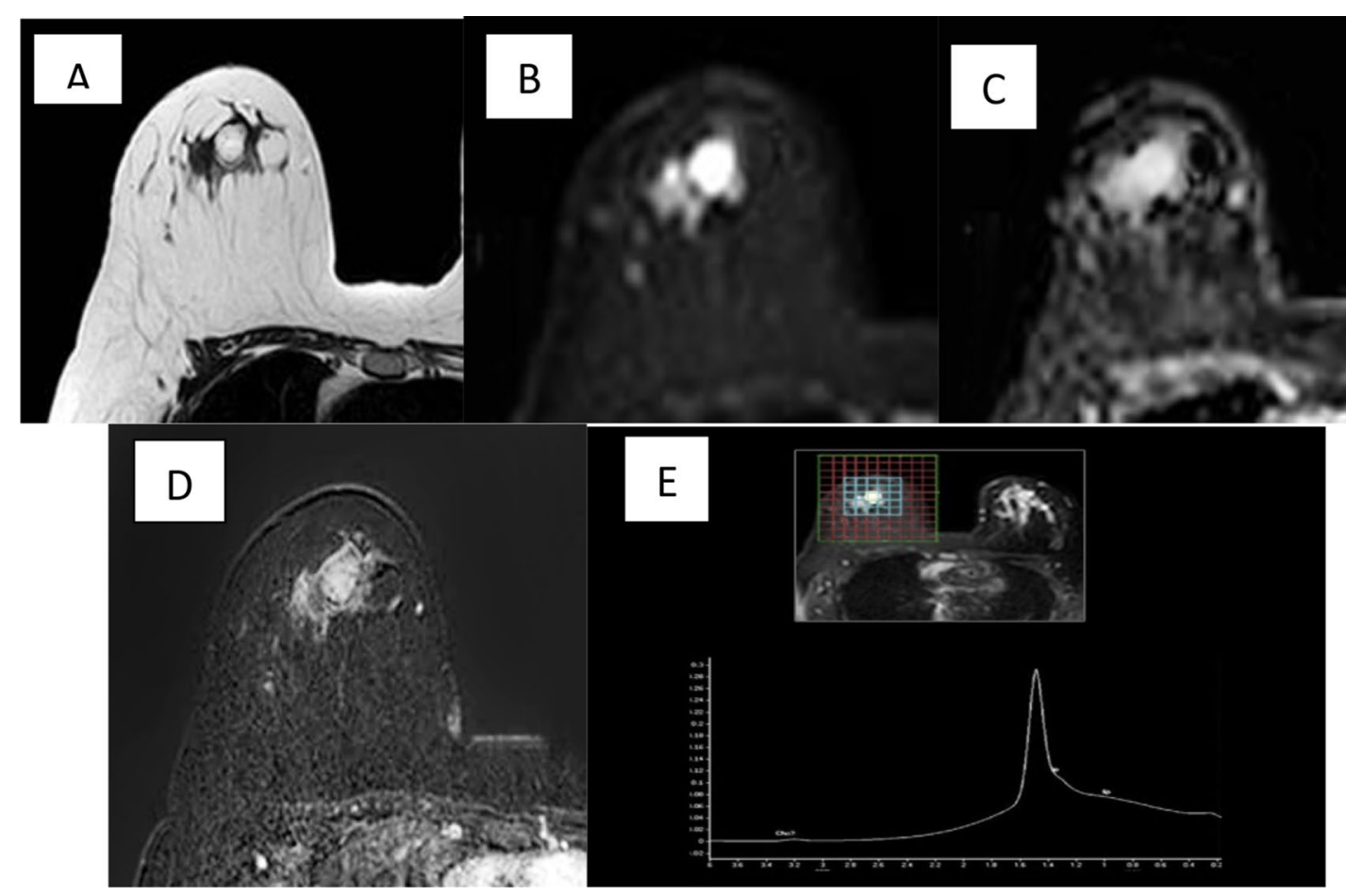

Fig. 3 A 37-year-old female with a right breast fibroadenoma. A Axial T2-WI, showing a well-circumscribed lesion of high signal with dark internal septations. B Axial DWI (b 1000), the lesion showed high signal. C Axial ADC map, the ADC value of the lesion was $1.25 \times 10^{-3} \mathrm{~mm}^{2} / \mathrm{s}$. D Axial postcontrast T1-WI with subtraction, the lesion had homogenous enhancement. E Multivoxel MRS study revealed negative choline peak

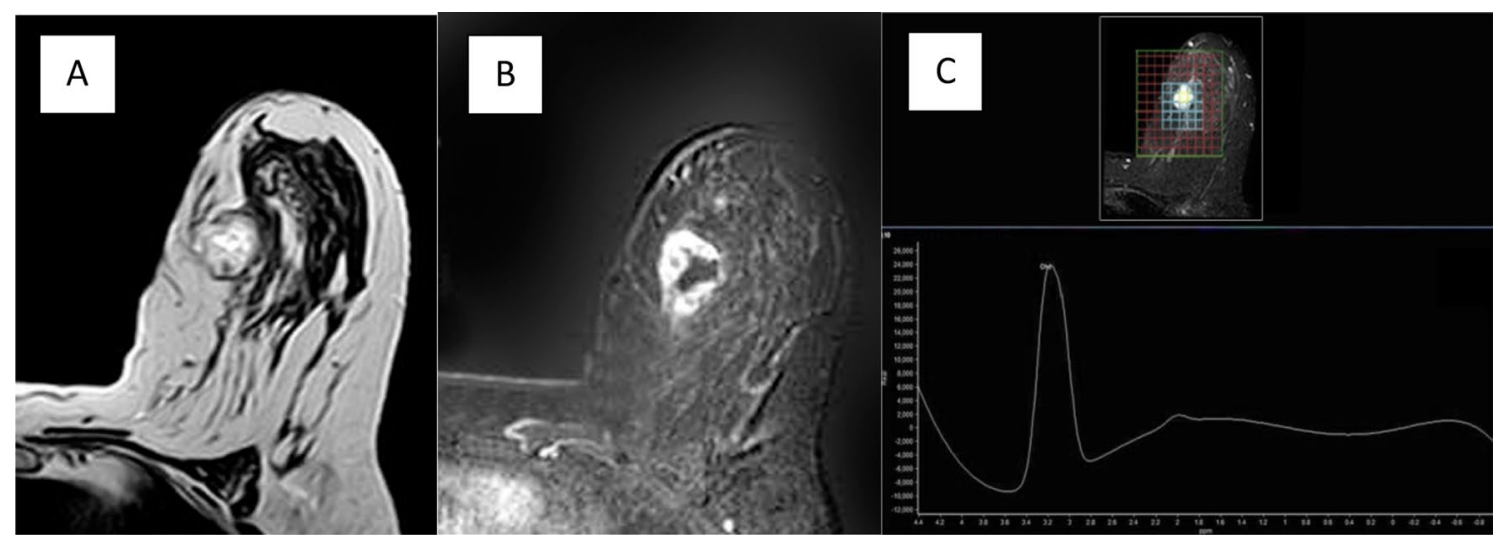

Fig. 4 A 49-year-old female with a left breast mucinous carcinoma. A Axial T2-WI showing a partially circumscribed lesion of mixed high signal. B Axial postcontrast T1-WI with subtraction, showing heterogeneous enhancement. C MRS study showing a positive choline peak 


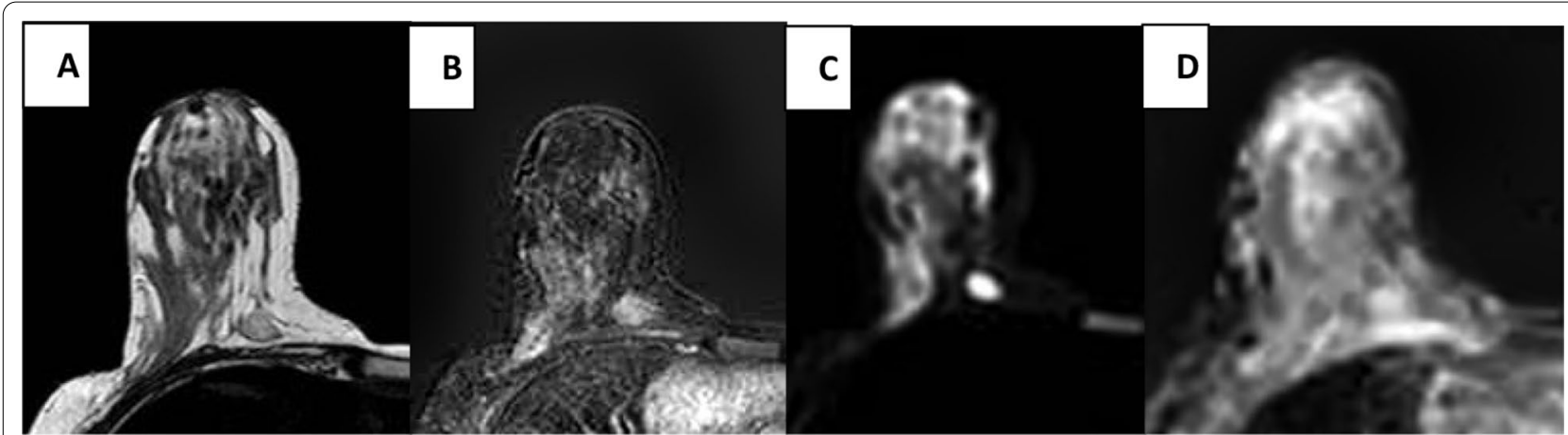

Fig. 5 A 35-year-old female with a right breast fibroadenoma. A Axial T2-WI showing a well-circumscribed lesion of low signal and dark internal septations. B Axial postcontrast T1-WI with subtraction, the lesion showed homogenous enhancement. C Axial DWI (b 1000), the lesion showed high signal. D Axial ADC map, the ADC value of the lesion was $1.09 \times 10^{-3} \mathrm{~mm}^{2} / \mathrm{s}$
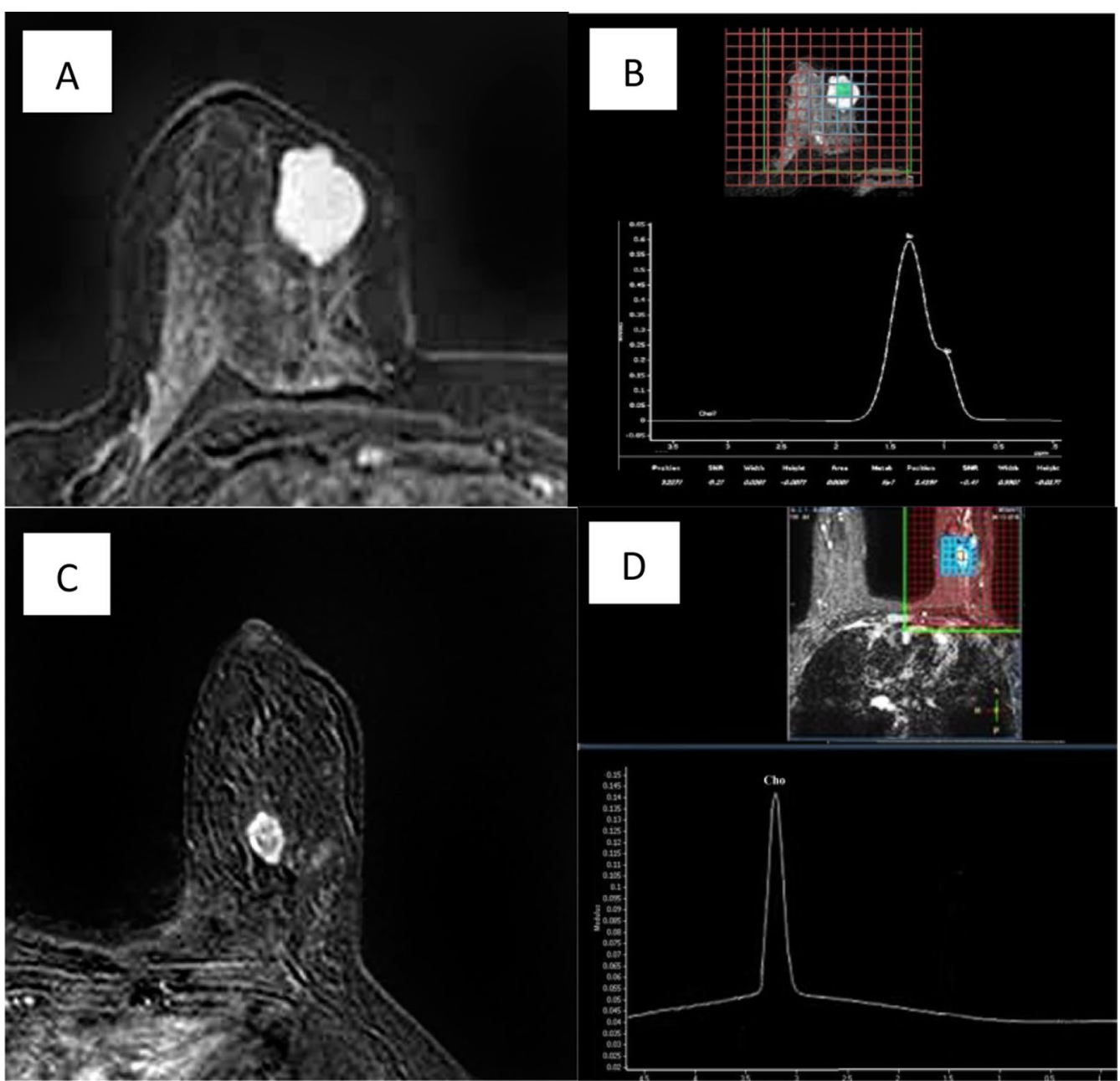

Fig. 6 Two different female patients: the first patient aged 36 years old and had right breast fibroadenoma. A Axial postcontrast T1-WI with subtraction, showing a well-circumscribed lobulated homogenously enhancing right breast mass. B MRS study showing absent choline peak. The second patient aged 42 years and had left breast mucinous carcinoma. C Axial postcontrast T1-WI with subtraction, showing a partially circumscribed heterogeneously enhancing left breast mass. D MRS study showing positive choline peak 


\section{Abbreviations}

DCE-MRI: Dynamic contrast-enhanced magnetic resonance imaging; MRI-BIRADS: MRI breast imaging reporting and data system; DWl: Diffusionweighted MR imaging; MRS: Multi-voxel MR spectroscopy; EPI: Echo planar imaging; ADC: Apparent diffusion coefficient.

\section{Acknowledgements}

Not applicable.

\section{Authors' contributions}

Dr. DB contributed to study design, data collection and manuscript preparation. Dr. AS performed in data collection and statistical analysis. Dr. EA was involved in data collection and manuscript revision. Dr. EA contributed to study design, data interpretation and manuscript writing. All authors have read and approved the manuscript.

\section{Funding}

No funding resources.

\section{Availability of data and materials Available.}

\section{Declarations}

\section{Ethics approval and consent to participate}

Ethics approval and consent to participate were approved and obtained by Mansoura Faculty of Medicine Institutional Research Board (MFM-IRB); reference number is not available. Informed consent was waived because this is a retrospective study.

\section{Consent for publication \\ Not applicable.}

\section{Competing interests}

The authors declare that they have no competing interests.

\section{Author details}

${ }^{1}$ Department of Diagnostic Radiology, Mansoura Faculty of Medicine, Mansoura University, Mansoura, Egypt. ${ }^{2}$ Department of General Surgery, Faculty of Medicine, Horus University, Damietta, Egypt.

Received: 28 June 2021 Accepted: 22 September 2021

Published online: 21 October 2021

\section{References}

1. Chaudhry AR, El Khoury M, Gotra A et al (2019) Imaging features of pure and mixed forms of mucinous breast carcinoma with histopathological correlation. Br J Radiol 92:20180810
2. Igarashi T, Ashida H, Morikawa K, Motohashi K, Fukuda K (2016) Use of BIRADS-MRI descriptors for differentiation between mucinous carcinoma and fibroadenoma. Eur J Radiol 85:1092-1098

3. Magnetic resonance imaging of fibroadenoma-like lesions and correlation with Breast Imaging-Reporting and Data System and Kaiser scoring system. SA J Radiol. ISSN: (Online) 2078-6778, (Print) 1027-202X. http:// www.sajr.org.za

4. Ferré R, Aldis A, AlSharif S (2016) Differentiation of fibroadenomas and pure mucinous carcinomas on dynamic contrast-enhanced MRI of the breast using volume segmentation for kinetic analysis: a feasibility study. AJR 206:253

5. Jagannathan NR, Sharma U (2017) Breast tissue metabolism by magnetic resonance spectroscopy. Metabolites 7:25

6. Sardanelli F, Carbonaro LA, Montemezzi S (2016) Clinical breast MR using MRS or DWl: who is the winner? Front Oncol 6:217

7. Morris E, Comstock C, Lee C et al (2013) Magnetic resonance imaging. In: Breast imaging reporting and data system. American College of Radiology, Reston, VA, ACR BI-RADS ${ }^{\circledR}$ atlas

8. Bitencourt AGV, Graziano L, Osrio CABT (2016) MRI features of mucinous cancer of the breast: correlation with pathologic findings and other imaging methods. AJR 206:238

9. Zhang L, Jia N, Han L et al (2015) Comparative analysis of imaging and pathology features of mucinous carcinoma of the breast. Clin Breast Cancer 15:e147-e154

10. Schnall J, Blume DA, Bluemke GAD, Angelis ND, Bruhl S, Harms S (2006) Diagnostic architectural and dynamic features at breast MR imaging: multicenter study. Radiology 238:42-53

11. Mahoney MC, Gatsonis C, Hanna L, De Martini WB, Lehman C (2012) Positive predictive value of BI-RADS MR imaging. Radiology 264:51-58

12. Kim SH, Cha ES, Kim HS et al (2009) Diffusion-weighted imaging of breast cancer: correlation of the apparent diffusion coefficient value with prognostic factors. J Magn Reson Imaging 30:615-620

13. Woodhams R, Kakita S, Hata H et al (2009) Diffusion-weighted imaging of mucinous carcinoma of the breast: evaluation of apparent diffusion coefficient and signal intensity in correlation with histologic findings. AJR Am J Roentgenol 193:260-266

14. Tan J, Xu L, Yao W et al (2015) In vivo post-contrast $1 \mathrm{H}-\mathrm{MRS}$ evaluation of malignant and benign breast lesions: a meta-analysis. Tumor Biol 36:345-352

15. Bayoumi D, Zak M, Abdallah Ibrahim D et al (2019) The additive role of $1 \mathrm{H}$-magnetic resonance spectroscopic imaging to ensure pathological complete response after neoadjuvant chemotherapy in breast cancer patients. Pol J Radiol 84:e570-e580

\section{Publisher's Note}

Springer Nature remains neutral with regard to jurisdictional claims in published maps and institutional affiliations.

\section{Submit your manuscript to a SpringerOpen ${ }^{\mathcal{O}}$ journal and benefit from:}

- Convenient online submission

- Rigorous peer review

- Open access: articles freely available online

- High visibility within the field

- Retaining the copyright to your article

Submit your next manuscript at $\boldsymbol{\nabla}$ springeropen.com 